\title{
Comparison of Absorbed Dose to Medium and Absorbed Dose to Water for Spine IMRT Plans Using a Commercial Monte Carlo Treatment Planning System
}

\author{
Muhammad Nauman Usmani ${ }^{{ }^{*}}$, Norihisa Masai ${ }^{2}$, Ryoong-Jin $\mathrm{Oh}^{2}$, Hiroya Shiomi ${ }^{2}$, \\ Daisaku Tatsumi ${ }^{2}$, Hideharu Miura ${ }^{2}$, Toshihiko Inoue $^{2}$, Masahiko Koizumi ${ }^{1}$ \\ ${ }^{1}$ Department of Medical Physics \& Engineering, Osaka University Graduate School of Medicine, \\ Osaka, Japan \\ ${ }^{2}$ Miyakojima IGRT Clinic, Osaka, Japan \\ Email: "naumanusmani@bzu.edu.pk
}

Received December 26, 2013; revised January 20, 2014; accepted February 7, 2014

Copyright (C 2014 Muhammad Nauman Usmani et al. This is an open access article distributed under the Creative Commons Attribution License, which permits unrestricted use, distribution, and reproduction in any medium, provided the original work is properly cited. In accordance of the Creative Commons Attribution License all Copyrights (C) 2014 are reserved for SCIRP and the owner of the intellectual property Muhammad Nauman Usmani et al. All Copyright @ 2014 are guarded by law and by SCIRP as a guardian.

\section{ABSTRACT}

Dose in radiation therapy has been reported as the water-equivalent dose using conventional dose calculation algorithms. The Monte Carlo (MC) algorithm employs characterization of human tissues by elemental composition and mass density. It enables more accurate dose calculation for radiation therapy treatment planning and typically reports absorbed dose to medium. Whether one should use dose to medium or tissue $\left(D_{m}\right)$ in place of dose to water $\left(D_{w}\right)$ for MC treatment planning remains the subject of debate. The aim of the current study is to evaluate the differences between dose-volume indices for $D_{m}$ and $D_{w}$ MC-calculated IMRT plans. Thirty-seven spine patients were selected for this study. The IMRT optimization and MC calculations were performed using the iPlan RT Dose ${ }^{\mathrm{TM}}$ ver 4.1.2 (Brainlab, Munich, Germany) treatment planning system (TPS) with an X-ray Voxel Monte Carlo (XVMC) dose calculation engine. $D_{w}$ and $D_{m}$ results for target and critical structures were evaluated using the dose-volume-based indices. Systematic differences between dose-volume indices computed with $D_{w}$ and $D_{m}$ were up to $5.2 \%, 4.2 \%$, and $4.5 \%$ for D2, D50 and D98 indices of the clinical target volume (CTV), respectively and up to $1 \%$ for the critical structure dose indices. Our study demonstrates that employing $D_{m}$ in place of $D_{w}$ in MC-calculated IMRT treatment plans introduces a significant systematic difference in target DVHs. We recommend that for diffused target structures (such as spine tumors), dose to water is a better quantity for dose prescription in photon beam treatment planning using existing MC TPS. While for critical structures, it would be reasonable to report $D_{m}$ always. However in future with the availability of finer spatial resolution, $\mathrm{D}_{\mathrm{m}}$ will be the most suitable variable for both target and critical structures' dose prescription and reporting in MC treatment planning.

\section{KEYWORDS}

Dose to Medium; Dose to Water; Monte Carlo; IMRT

\section{Introduction}

Conventional dose calculations for photon beam radiation therapy including both simple correction-based algorithms and model-based algorithms typically report the absorbed dose to water, $\mathrm{D}_{\mathrm{w}}$ i.e. energy absorbed in a small cavity of water divided by the mass of that cavity.

${ }^{*}$ Corresponding author.

This reporting in terms of $D_{w}$ is due partly to the historical development of treatment planning algorithms as well as the fact that accelerator and ionization chamber calibration protocols are based on $D_{w}$ [1-3]. The input data used for treatment-planning system (TPS) commissioning are generally dose profiles and output factors measured in water phantoms. The assumption that the patient body is water is a good first approximation as water 
makes up the bulk of the volume of cells and body fluid and most biological tissues (with a few exceptions such as compact bone, tooth enamel and lung) have radiation properties similar to those of water [4-6].

Instead of treating human tissues as water of various densities as in analytical algorithms, the Monte Carlo (MC) algorithm employs characterization of human tissues by elemental composition and mass density. Consequently, it provides the most accurate dose calculations allowing all material specific electromagnetic and nuclear interactions [6-9]. Since particle transport simulations occur in materials representative of patient media, MC dose calculation engines calculate dose to medium or tissue $\left(\mathrm{D}_{\mathrm{m}}\right)$ i.e. energy absorbed in a small tissue element divided by the mass of tissue element [10-15].

In order to compare $\mathrm{MC} \mathrm{D}_{\mathrm{m}}$ algorithms with conventional $D_{w}$ algorithms, a method to convert $D_{m}$ to $D_{w}$ is required. Siebers et al. developed a procedure that converts $D_{m}$ to $D_{w}$ using stopping power ratios, based upon the Bragg-Gray cavity theory for MC-based calculations [16].

As one has to compromise the accuracy and information of the MC results by converting them to $D_{w}$, most physicists believe that $D_{m}$ is the preferred parameter for treatment planning, and that it should replace $D_{w}$. Whether one should use $D_{m}$ in place of $D_{w}$ directly in clinical prescriptions remains the subject of debate and there are strong arguments both for using $\mathrm{D}_{\mathrm{m}}$ or $\mathrm{D}_{\mathrm{w}}$.

Different clinical calculation methods may yield different quantities related to the absorbed dose for a given tissue and any significant differences between $D_{w}$ and $D_{m}$ might lead to the change of dose prescription [4,17-19]. A clinical decision has to be made during radiotherapy treatment planning as to whether one should prescribe the dose to the target volume that contains different biological media using $\mathrm{D}_{\mathrm{m}}$ or the converted $\mathrm{D}_{\mathrm{w}}[20]$.

Studies comparing $D_{m}$ with $D_{w}$ have been conducted for photon dose calculations. It has been shown that for soft tissues the difference may be in the order of $1 \%-2 \%$ $[16,21]$. However, for higher density materials, such as cortical bone, the difference can be as large as $15 \%$. Dogan et al. demonstrated that converting $\mathrm{D}_{\mathrm{m}}$ to $\mathrm{D}_{\mathrm{w}}$ in MC-calculated IMRT plans introduces a systematic error of up to $5.8 \%$ for head and neck tumors and $8.0 \%$ for prostate cases [19].

It is worth mentioning that the reported clinical data about $\mathrm{D}_{\mathrm{m}}$ and $\mathrm{D}_{\mathrm{w}}$ are limited to a few tumour sites. In addition, to the best of our knowledge, the comparative studies for $D_{m}$ and $D_{w}$ using a commercial MC TPS have not been conducted. As MC-calculation algorithms are being introduced into routine clinical practice [22-26], it has become increasingly important to know how much $D_{w}$ and $D_{m}$ differ in order to determine the significance of this conversion for different clinical cases. The pur- pose of the present study was to evaluate the dose differences in target and critical structures for $\mathrm{D}_{\mathrm{m}^{-}}$and $\mathrm{D}_{\mathrm{w}}$-based spine IMRT plans using a commercial MC TPS.

\section{Materials \& Methods}

\subsection{Treatment Plan Selection}

Thirty seven patients treated for spine tumor with IMRT using the Novalis $^{\mathrm{TM}}$ shaped beam radiosurgery unit (Brainlab, Munich, Germany) were selected for this study. Patient characteristics (Age, Gender, CTV, and Tumor location) are shown in Table 1.

\subsection{Treatment Planning}

3D-CT scans were performed on a 4-slice Brightspeed QX/i scanner (GE Medical Systems, Waukesha, WI, USA) with the patient in a supine position and with arms raised above the head. Immobilization during simulation and subsequent treatment was achieved by using a Vacuum-Type immobilization and Thermo Plastic type device. The CT images were acquired with a slice thickness and spacing of $1.25 \mathrm{~mm}$ and with gantry rotation time of 1.0 second. $6 \mathrm{MV}$ photon beams were used for the IMRT treatment planning. For the spine cases evaluated in this work, whole vertebra was delineated as CTV. The PTV was generated by adding a 3-mm margin to CTV and the isocenter was positioned at the center of the PTV. The critical structures included the spinal cord, esophagus, trachea and lung depending on the tumor location.

Doses of 40 - 72 Gy delivered in 5 to 20 fractions, equivalent to BED10 $(\alpha / \beta=10)(\max =107.1 \mathrm{~Gy}, \min =$ $65.5 \mathrm{~Gy}$, median $=78 \mathrm{~Gy}$ ) were prescribed with the following planning objectives:

PTV: D95\% > 95\% and V95\% > 95\%, i.e. 95\% of PTV volume should receive at least $95 \%$ of the prescribed dose; Spinal cord: D0.1 cc < 100 Gy (BED2 $(\alpha / \beta$ $=2)$ ), i.e. a volume receiving 100 Gy (BED2) should be less than 0.1 cc to avoid radiation myelopathy [27].

Multiple coplanar and non-opposing beams were utilized with different angles depending on the tumor

Table 1. Patient characteristics.

\begin{tabular}{cc}
\hline Patient (n) & 37 \\
\hline Age (y) & $68(34-85)$ \\
Males: Females & $20: 17$ \\
Clinical target volume (cc) & $73.14(13.36-154.0)$ \\
Target location & 21 \\
Upper Thoracic (Th1 - Th6) (n) & 16 \\
Lower Thoracic (Th7 - Th12) (n)
\end{tabular}


location. The IMRT optimization and MC calculations were performed using the iPlan RT Dose ${ }^{\mathrm{TM}}$ ver 4.1.2 (Brainlab, Munich, Germany) TPS with an X-ray Voxel Monte Carlo (XVMC) dose calculation engine.

A nominal value of $1 \%$ of the maximum dose mean variance per beam was used for all MC dose calculations, leading to a $<1 \%$ statistical uncertainty in the dose to the targeted tissues. The spatial resolution was set at $2.0 \mathrm{~mm}$ for all calculations. It has been shown that an overall $2 \%$ statistical uncertainty has minimal effect on DVHs, and these statistical values are adequate for dose-volume analysis [28,29].

\subsection{Plan Analysis}

The MC-calculated $\mathrm{D}_{\mathrm{m}}$ - and $\mathrm{D}_{\mathrm{w}}$-based plans were evaluated quantitatively using the dose-volume-based indices: doses received by $2 \%, 50 \%$ and $98 \%$ of the target volumes (D2, D50, and D98), doses received by $2 \%$ of the critical structure volumes (D2), and doses to the $0.1 \mathrm{cc}$, $0.5 \mathrm{cc}$, and $1 \mathrm{cc}$ (D0.1 cc, D0.5 cc, and D1 cc) of the critical structures. The mean, standard deviation and range of percent differences in dose-volume indices were calculated. The $\mathrm{D}_{\mathrm{m}}$ - and $\mathrm{D}_{\mathrm{w}}$-based isodose distributions and DVHs for one of the cases are presented to demonstrate the significant differences. The differences between $\mathrm{D}_{\mathrm{m}}$ - and $\mathrm{D}_{\mathrm{w}}$-based dose distributions are also calculated using an in-house developed system.

\section{Results}

Our study describes the differences of the MC-calculated IMRT plans using $D_{w}$ - and $D_{m}$-based evaluation for thirty seven spine tumor patients. The isodose distributions through a transverse patient slice and DVHs for one of the IMRT plans are shown in Figure 1. As shown in Figures 1(a) and (b), the $110 \%$ isodose line is missing in $\mathrm{D}_{\mathrm{m}}$ plan, while it covers a considerable part of the target in $\mathrm{D}_{\mathrm{w}}$ plan. The $108 \%, 106 \%, 104 \%$ and $102 \%$ lines also vary noticeably between the two dose distributions. This isodose line shift is due to the fact that the target tissues infiltrate the high density bone content of vertebra. Figure 1(c) shows that employing $D_{m}$ in place of $D_{w}$ shifts the resulting DVHs for the target volumes by about $5 \%$, and for the critical structures by about $1 \%$. Figure 2 shows the MC-based $D_{w}$ and $D_{m}$ differences expressed in terms of isodose distributions and DVH for the same patient plan. A maximum difference of $\sim 11 \%$ can be observed on the scale of $100 \%=3 \mathrm{~Gy}$.

The ranges of percent differences in dose-volume indices, evaluated for $D_{w}$ and $D_{m}$ for thirty seven spine cases, are summarized in Table 2. Figure 3(a) shows the variations of the D2, D50 and D98 indices of the CTV in terms of $D_{w} / D_{m}$ for all patients. In all cases, the systematic differences between $\mathrm{D}_{\mathrm{w}}$ - and $\mathrm{D}_{\mathrm{m}}$-based $\mathrm{D} 2$ ranged

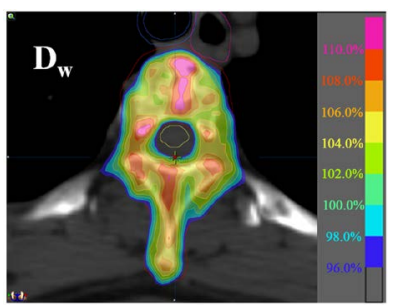

(a)

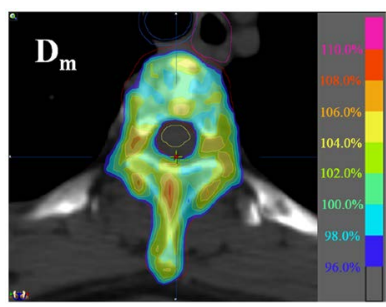

(b)

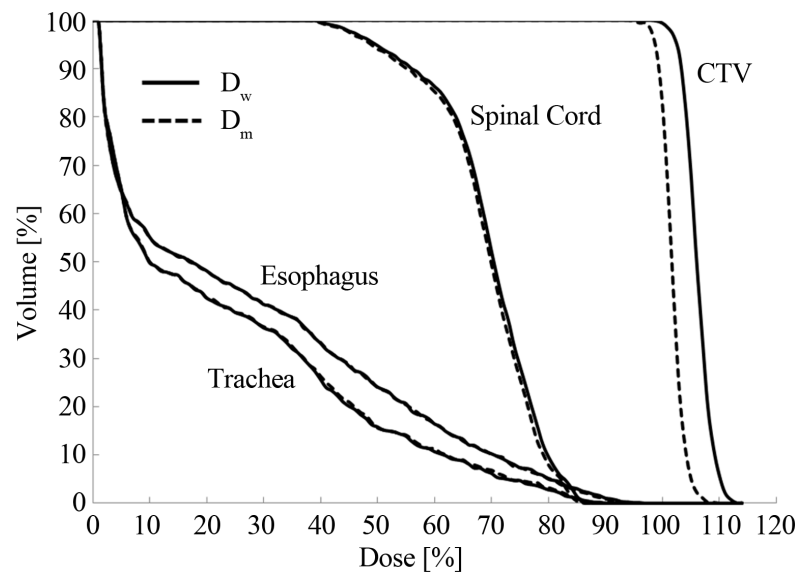

(c)

Figure 1. The MC-based dose-to-water $\left(D_{w}\right)$ and dose-tomedium $\left(D_{m}\right)$ results for one of the spine patient plans: (a) $D_{w}$; (b) $D_{m}$ and (c) DVH comparison.

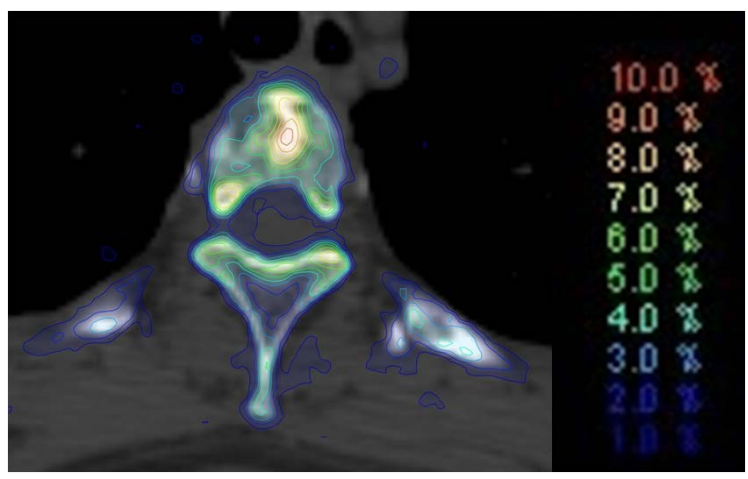

(a)

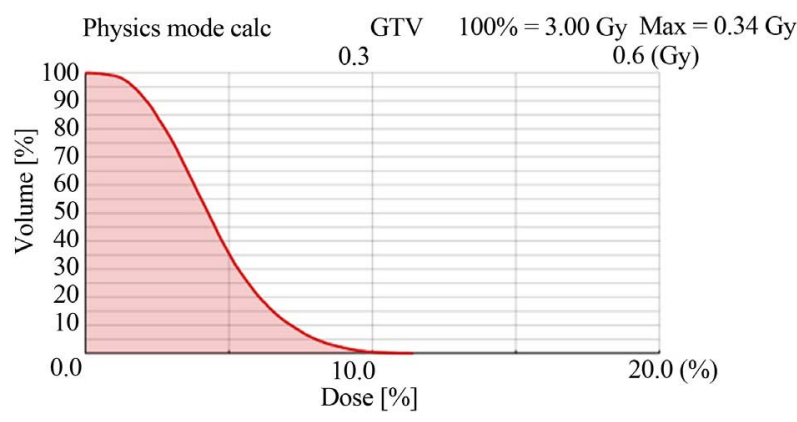

(b)

Figure 2. The differences between dose-to-water $\left(D_{w}\right)$ and dose-to-medium $\left(D_{m}\right)$ expressed in terms of $(a)$ isodose distributions and (b) DVH for the same patient plan. 


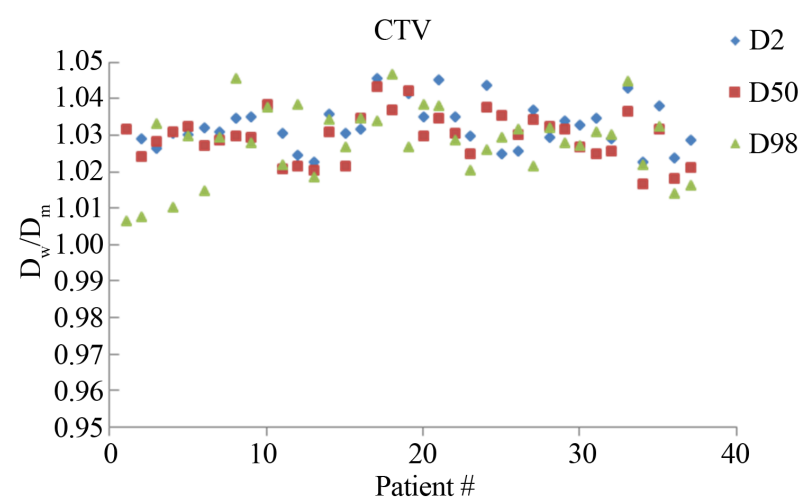

(a)

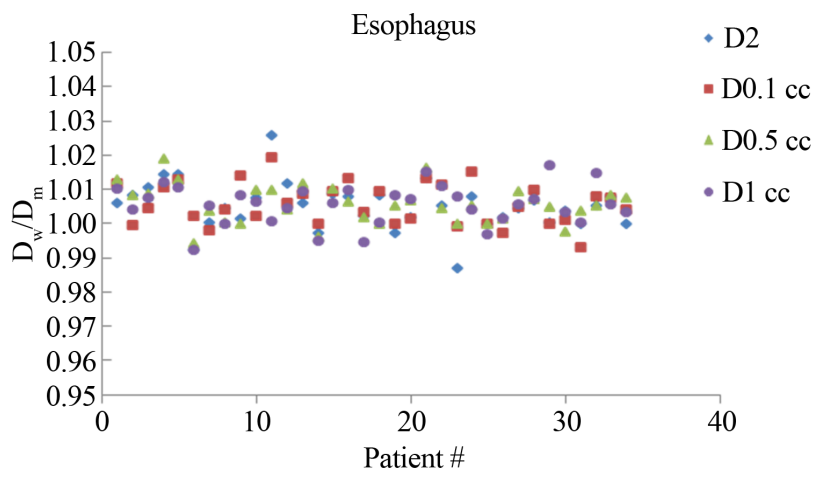

(c)

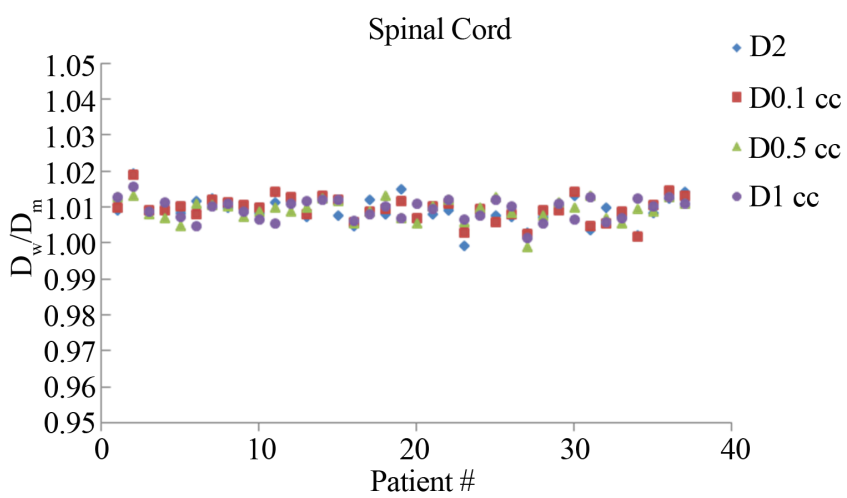

(b)

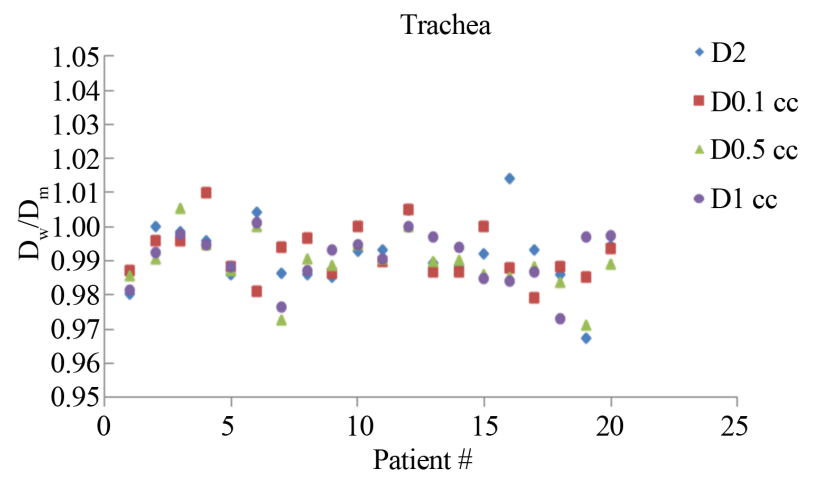

(d)

Figure 3. Variations of $D_{w} / D_{m}$ of (a) D2, D50 and D98 of CTV, D2, D0.1 cc, D0.5 cc and D1 cc of (b) Spinal cord; (c) Esophagus and (d) Trachea.

Table 2. The mean (standard deviation) and range of percent differences in dose-volume indices evaluated for $D_{w}$ and $D_{m}$ for thirty seven spine IMRT cases. Numbers in square brackets indicate the range.

\begin{tabular}{|c|c|c|c|c|c|c|c|}
\hline Structure & $\begin{array}{l}\left(\frac{D_{w}^{98}-D_{m}^{98}}{D_{w}^{98}}\right) \\
\times 100(\%)\end{array}$ & $\begin{array}{l}\left(\frac{D_{w}^{50}-D_{m}^{50}}{D_{w}^{50}}\right) \\
\times 100(\%)\end{array}$ & $\begin{array}{l}\left(\frac{D_{w}^{2}-D_{m}^{2}}{D_{w}^{2}}\right) \\
\times 100(\%)\end{array}$ & $\begin{array}{l}\left(\frac{D_{w}^{0.1 c c}-D_{m}^{0.1 c c}}{D_{w}^{0.1 c c}}\right) \\
\times 100(\%)\end{array}$ & $\begin{array}{l}\left(\frac{D_{w}^{0.5 c c}-D_{m}^{0.5 c c}}{D_{w}^{0.5 c c}}\right) \\
\times 100(\%)\end{array}$ & $\begin{array}{l}\left(\frac{D_{w}^{1.0 c c}-D_{m}^{1.0 c c}}{D_{w}^{1.0 c c}}\right) \\
\times 100(\%)\end{array}$ & $\begin{array}{l}\left(\frac{D_{w}^{5.0 c c}-D_{m}^{5.0 c c}}{D_{w}^{5.0 c c}}\right. \\
\times 100(\%)\end{array}$ \\
\hline CTV & $\begin{array}{l}2.7(0.9) \\
{[0.7-4.5]}\end{array}$ & $\begin{array}{l}2.9(0.6) \\
{[1.7-4.2]}\end{array}$ & $\begin{array}{l}3.2(0.7) \\
{[2.2-5.2]}\end{array}$ & - & - & - & - \\
\hline Spinal Cord & - & - & $\begin{array}{c}0.9(0.4) \\
{[0.0-1.9]}\end{array}$ & $\begin{array}{c}0.9(0.4) \\
{[0.2-1.9]}\end{array}$ & $\begin{array}{c}0.9(0.3) \\
{[-0.1-1.3]}\end{array}$ & $\begin{array}{l}0.9(0.3) \\
{[0.1-1.6]}\end{array}$ & - \\
\hline Esophagus & - & - & $\begin{array}{c}0.5(0.7) \\
{[-1.3-2.5]}\end{array}$ & $\begin{array}{c}0.6(0.6) \\
{[-0.70-1.9]}\end{array}$ & $\begin{array}{c}0.6(0.6) \\
{[-0.6-1.9]}\end{array}$ & $\begin{array}{c}0.5(0.6) \\
{[-0.7-1.67]}\end{array}$ & $\begin{array}{c}0.2(0.7) \\
{[-2.0-1.4]}\end{array}$ \\
\hline Trachea & - & - & $\begin{array}{c}-0.8(1.0) \\
{[-3.4-1.4]}\end{array}$ & $\begin{array}{l}-0.8(0.8) \\
-2.1-0.9]\end{array}$ & $\begin{array}{c}-1.1(0.8) \\
{[-2.9-0.5]}\end{array}$ & $\begin{array}{c}-0.9(0.8) \\
{[-2.7-0.1]}\end{array}$ & $\begin{array}{l}-1.0(0.8) \\
{[-2.9-0]}\end{array}$ \\
\hline
\end{tabular}

from $2.2 \%$ to $5.2 \%$ with an average of $3.2 \%$. For all patients, the systematic differences between $\mathrm{D}_{\mathrm{w}^{-}}$and $\mathrm{D}_{\mathrm{m}^{-}}$ based CTV D50 ranged from $1.7 \%$ to $4.2 \%$ with an average of $2.9 \%$. The systematic differences between $\mathrm{D}_{\mathrm{w}}$ and $\mathrm{D}_{\mathrm{m}}$-based CTV D98 ranged from $0.7 \%$ to $4.5 \%$ with an average of $2.7 \%$ for all cases. The large systematic shift is attributed to high bone content in the CTV. Figure 3(b) shows the variations of the $\mathrm{D}_{\mathrm{w}^{-}}$and $\mathrm{D}_{\mathrm{m}}{ }^{-}$ based D2, D0.1 cc, D0.5 cc and D1 cc indices of the spinal cord for all patients. The average systematic differences were within $0.9 \%$. This small difference is due to the fact that spinal cord consists of soft tissues with no direct interfaces with bone.

Figure $3(c)$ shows the variations of the $D_{w^{-}}$and $D_{m^{-}}$ based D2, D0.1 cc, D0.5 cc, and D1 cc indices of the esophagus for thirty five patients. The average systematic differences were within $0.6 \%$. This minimal difference can be attributed to the cumulative effect of air-soft tissue and bone-soft tissue interfaces. Figure 3(d) shows the variations of the $\mathrm{D}_{\mathrm{w}}$ - and $\mathrm{D}_{\mathrm{m}}$-based D2, D0.1 cc, D0.5 cc, and D1 cc indices of the trachea for twenty patients. The average systematic differences were within $-1.1 \%$. 
This negative difference is due to the air-soft tissue interfaces all around the trachea.

Figure 4 shows the $D_{w} / D_{m}$ as a function of the mean CT value of CTV. With a p-value $<0.05$, a significant linear correlation between $\mathrm{D}_{\mathrm{w}} / \mathrm{D}_{\mathrm{m}}$ and mean $\mathrm{CT}$ value can be observed.

Note that for CTV, the wide ranges of dose-volume indices may be attributed to the variations in physical density. For high mean CT values, the absorbed dose to water is generally high. Nevertheless, it might also depend on treatment plan and delivery. Wide ranges of dose-volume indices for esophagus and trachea are due to the fact that for different cases, these structures have dissimilar interfaces with surrounding organs. However, for all cases spinal cord has nearly identical surroundings, resulting in narrow ranges of dose-volume indices.

\section{Discussion}

Traditionally, $\mathrm{D}_{\mathrm{w}}$ has been reported for dose computations in radiation therapy with high-energy photon beams. Treatment planning employing MC techniques allows the radiation transport and energy deposition in patient representative media and the absorbed dose reported in this process can be either the $D_{m}$, or the $D_{w}$ [30].

Whether one should use $D_{w}$ or $D_{m}$ for MC treatment planning is still a controversial topic. Supporters of $D_{m}$ claim that dose to the tissues of interest is the quantity inherently computed by MC dose algorithms and the rationale for converting $D_{m}$ back to $D_{w}$ is driven solely by the desire to comply with tradition. They argue that there is an increased uncertainty and complexity arising from the introduction of an additional quantity for calculating $D_{w}[16,20]$. They also state that the clinical impact of switching from $D_{w}$ to $D_{m}$ is not expected to be significant, mainly because most tissues of interest in radiotherapy are similar to water. On the other hand, those who advocate for the usage of $D_{w}$ argue that all historical clinical experience and modern dosimetry protocols are

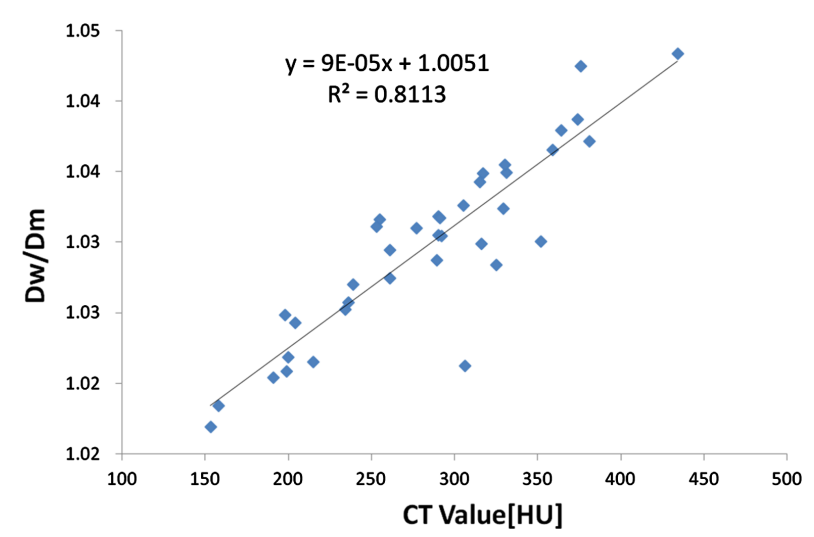

Figure 4. $D_{w} / D_{m}$ as a function of the mean CT value of CTV. The line shows a linear fit through the data.
$\mathrm{D}_{\mathrm{w}}$-based and the medium employed to report the absorbed dose is always uncertain because the exact composition is not known for real patients $[3,18,20]$. They further state that tumor cells embedded within a medium are more water-like than medium-like and may thus be modelled as a water cavity within the medium. The AAPM TG 105 report recommends that TPS make both $\mathrm{D}_{\mathrm{w}}$ and $\mathrm{D}_{\mathrm{m}}$ options available for dose reporting [6].

Ma et al. demonstrated that conventional photon dose calculation algorithms compute doses using water with different electron densities, which are close ( $<4 \%$ differences) to doses to media, as computed by MC, but significantly different (up to 11\%) from doses to water converted from doses to media [30]. They suggested that for consistency with previous radiation therapy experience, MC photon algorithms report dose to medium for radiotherapy dose prescription, treatment plan evaluation and treatment outcome analysis. Walters et al. suggested that it is better to specify $D_{w}$ than $D_{m}$ in MC treatment plans since $D_{w}$ provides a better estimate of dose to sensitive skeletal tissue [31].

To appraise the significance of $D_{m}$ to $D_{w}$ conversion, we evaluated the differences between $D_{w}$ - and $D_{m}$-based MC dose calculations for a large set of clinical cases. In this work, the MC algorithm was used to calculate the $D_{w}$ and $\mathrm{D}_{\mathrm{m}}$ for all of the plans using a commercial TPS. Our study shows that conversion from $\mathrm{D}_{\mathrm{m}}$ to $\mathrm{D}_{\mathrm{w}}$ in MC-calculated spine IMRT treatment plans introduces significant differences in target DVHs ranged from $2.21 \%$ to $5.18 \%$. For critical structures, however, the average differences between $D_{w}$ and $D_{m}$ are within $1 \%$.

Dose to water is substantially larger compared to dose to medium for tumor cells infiltrated in bony tissues. This is due to the fact that high density bone causes a higher fluence of secondary electrons in the water cavity and consequently a higher dose is deposited compared to the case of the cavity filled also with bone. The dose to water should be selected for soft tissue cells within a bony structure and dose to medium is a better option to know the average dose within the whole voxel.

The aim of the prescribed dose is to deliver a lethal dose to the tumor cells with a retrievable damage to the normal cells embedded in the tumor. $\mathrm{D}_{\mathrm{w}}$-based treatment planning yields to the clinical prescription for the cells embedded in heterogeneous tissues such as lung or bone.

The CT number to medium-type conversion has a significant uncertainty due to CT partial volume effect and the mixture of biological tissues such as air, soft tissue and compact bone inside a dose calculation voxel $[6,8$, 31-34]. For cells embedded in heterogeneous tissues, accuracy of computed dose is strongly affected by the size of internal MC dose computation grid.

We have been using $\mathrm{D}_{\mathrm{m}}$ in $\mathrm{MC}$ treatment planning for all tumor sites as routine clinical practice. However, 
based on our results and above discussion we recommend that for diffused target structures (such as spine tumors), dose to water is a better quantity for dose prescription in photon beam treatment planning using currently available MC TPS. While for critical structures, it would be reasonable to report $\mathrm{D}_{\mathrm{m}}$ always.

There is also an issue of selecting a better quantity for future radiotherapy dose prescription and reporting. With the innovation of advanced technologies in radiotherapy, if finer spatial resolution is made available, i.e. the size of the dose computation grid is significantly reduced to recognize the subvoxel structures, $D_{m}$ will be the most suitable and natural approach for both target and critical structures' dose prescription and reporting in MC treatment planning.

\section{Conclusion}

In this study, we have investigated the differences between $\mathrm{D}_{\mathrm{w}^{-}}$and $\mathrm{D}_{\mathrm{m}}$-based spine IMRT plans using a commercial MC TPS. Our data shows that for the target cells with a diffused pattern in bony anatomy, dose to water can be higher by $\sim 5 \%$ compared to dose to medium. Our results suggest that dose to water is more appropriate for dose prescription in target cells embedded in heterogeneous tissues using current photon beam MC TPS. However, in future with finer spatial resolution available, $D_{m}$ will be the preferred option to achieve the greatest accuracy in dose calculation for both target and critical structures.

\section{Acknowledgements}

This work was supported by a JSPS Core-to-Core program from the Japan Society for the Promotion of Science (Grant No. 23003).

\section{Conflict of Interest}

None.

\section{REFERENCES}

[1] P. R. Almond, P. J. Biggs, B. M. Coursey, et al., “AAPM’s TG-51 Protocol for Clinical Reference Dosimetry of High-Energy Photon and Electron Beams,” Medical Physics, Vol. 26, No. 9, 1999, pp. 1847-1870. http://dx.doi.org/10.1118/1.598691

[2] M. S. Huq, P. Andreo and H. Song, "Comparison of the IAEA TRS-398 and AAPM TG-51 Absorbed Dose to Water Protocols in the Dosimetry of High-Energy Photon and Electron Beams,” Physics in Medicine \& Biology, Vol. 46, No. 11, 2001, pp. 2985-3006. http://dx.doi.org/10.1088/0031-9155/46/11/315

[3] J. V. Siebers, P. J. Keall, A. E. Nahum and R. Mohan, "Converting Absorbed Dose to Medium to Absorbed Dose to Water for Monte Carlo Based Photon Beam Dose
Calculations,” Physics in Medicine \& Biology, Vol. 45, No. 4, 2000, pp. 983-995.

http://dx.doi.org/10.1088/0031-9155/45/4/313

[4] C. M. Ma, T. Pawlicki, S. B. Jiang, et al., "Monte Carlo Verification of IMRT Dose Distributions from a Commercial Treatment Planning Optimization System," Physics in Medicine \& Biology, Vol. 45, No. 9, 2000, pp. 2483-2495. http://dx.doi.org/10.1088/0031-9155/45/9/303

[5] H. H. Liu, "Dm Rather than Dw Should Be Used in Monte Carlo Treatment Planning. For the Proposition,” Medical Physics, Vol. 29, No. 5, 2002, pp. 922-923. http://dx.doi.org/10.1118/1.1473137

[6] I. J. Chetty, B. Curran, J. E. Cygler, et al., "Report of the AAPM Task Group No. 105: Issues Associated with Clinical Implementation of Monte Carlo-Based Photon and Electron External Beam Treatment Planning," Medical Physics, Vol. 34, No. 12, 2007, pp. 4818-4853. http://dx.doi.org/10.1118/1.2795842

[7] F. Verhaegen and J. Seuntjens, "Monte Carlo Modelling of External Radiotherapy Photon Beams," Physics in Medicine \& Biology, Vol. 48, No. 21, 2003, pp. R107R164. http://dx.doi.org/10.1088/0031-9155/48/21/R01

[8] C. M. Ma and S. B. Jiang, "Monte Carlo Modelling of Electron Beams from Medical Accelerators," Physics in Medicine \& Biology, Vol. 44, No. 12, 1999, pp. R157R189. http://dx.doi.org/10.1088/0031-9155/44/12/201

[9] P. Andreo, "Monte Carlo Techniques in Medical Radiation Physics," Physics in Medicine \& Biology, Vol. 36, No. 7, 1991, pp. 861-920. http://dx.doi.org/10.1088/0031-9155/36/7/001

[10] T. D. Solberg, J. J. DeMarco, F. E. Holly, J. B. Smathers and A. A. DeSalles, "Monte Carlo Treatment Planning for Stereotactic Radiosurgery,” Radiotherapy \& Oncology, Vol. 49, No. 1, 1998, pp. 73-84. http://dx.doi.org/10.1016/S0167-8140(98)00065-6

[11] J. S. Li, G. M. Freedman, R. Price, et al., "Clinical Implementation of Intensity-Modulated Tangential Beam Irradiation for Breast Cancer,” Medical Physics, Vol. 31, No. 5, 2004, pp. 1023-1031. http://dx.doi.org/10.1118/1.1690195

[12] L. Jin, L. Wang, J. Li, W. Luo, S. J. Feigenberg and C. M. Ma, "Investigation of Optimal Beam Margins for Stereotactic Radiotherapy of Lung-Cancer Using Monte Carlo Dose Calculations,” Physics in Medicine \& Biology, Vol. 52, No. 12, 2007, pp. 3549-3561. http://dx.doi.org/10.1088/0031-9155/52/12/014

[13] E. Sterpin, F. Salvat, R. Cravens, K. Ruchala, G. H. Olivera and S. Vynckier, "Monte Carlo Simulation of Helical Tomotherapy with PENELOPE,” Physics in Medicine \& Biology, Vol. 53, No. 12, 2008, pp. 2161-2180. http://dx.doi.org/10.1088/0031-9155/53/8/011

[14] K. Bush, R. Townson and S. Zavgorodni, "Monte Carlo Simulation of RapidArc Radiotherapy Delivery,” Physics in Medicine \& Biology, Vol. 53, No. 19, 2008, pp. N359N370. http://dx.doi.org/10.1088/0031-9155/53/19/N01

[15] J. V. Siebers, P. J. Keall, J. O. Kim and R. Mohan, “A Method for Photon Beam Monte Carlo Multileaf Collimator Particle Transport," Physics in Medicine \& Biology, Vol. 47, No. 17, 2002, pp. 3225-3249. 
http://dx.doi.org/10.1088/0031-9155/47/17/312

[16] J. V. Siebers, P. J. Keall, A. E. Nahum and R. Mohan, "Converting Absorbed Dose to Medium to Absorbed Dose to Water for Monte Carlo Based Photon Beam Dose Calculation," Physics in Medicine \& Biology, Vol. 45, No. 4, 2000, pp. 983-995. http://dx.doi.org/10.1088/0031-9155/45/4/313

[17] F. C. Du Plessis, C. A. Willemse, M. G. Lötter and L. Goedhals, “The Indirect Use of CT Numbers to Establish Material Properties Needed for Monte Carlo Calculation of Dose Distributions in Patients," Medical Physics, Vol. 25, No. 7, 1998, pp. 1195-1201. http://dx.doi.org/10.1118/1.598297

[18] M. Fippel and F. Nüsslin, "Comments on 'Converting Absorbed Dose to Medium to Absorbed Dose to Water for Monte Carlo Based Photon Beam Dose Calculations',' Physics in Medicine \& Biology, Vol. 45, No. 8, 2007, pp. L17-L19. http://dx.doi.org/10.1088/0031-9155/45/8/101

[19] N. Dogan, J. V. Siebers and P. J. Keall, "Clinical Comparison of Head and Neck and Prostate IMRT Plans Using Absorbed Dose to Medium and Absorbed Dose to Water," Physics in Medicine \& Biology, Vol. 51, No. 19, 2006, pp. 4967-4980. http://dx.doi.org/10.1088/0031-9155/51/19/015

[20] P. Keall, "Dm Rather than Dw Should Be Used in Monte Carlo Treatment Planning. Against the Proposition," Medical Physics, Vol. 29, No. 5, 2002, pp. 923-924.

[21] C. M. Ma, E. Mok, A. Kapur, et al., "Clinical Implementation of a Monte Carlo Treatment Planning System," Medical Physics, Vol. 26, No. 10, 1999, pp. 2133-2143. http://dx.doi.org/10.1118/1.598729

[22] J. J. DeMarco, T. D. Solberg and J. B. Smathers, “A CTBased Monte Carlo Simulation Tool for Dosimetry Planning and Analysis," Medical Physics, Vol. 25, No. 1, 1998, pp. 1-11. http://dx.doi.org/10.1118/1.598167

[23] T. D. Solberg, J. J. DeMarco, F. E. Holly, J. B. Smathers and A. A. F. DeSalles, "Monte Carlo Treatment Planning for Stereotactic Radiosurgery,” Radiotherapy \& Oncology, Vol. 49, No. 1, 1998, pp. 73-84.

http://dx.doi.org/10.1016/S0167-8140(98)00065-6

[24] L. Wang, C. S. Chui and M. Lovelock, "A Patient-Specific Monte Carlo Dose-Calculation Method for Photon Beams,” Medical Physics, Vol. 25, No. 6, 1998, pp. 867-878. http://dx.doi.org/10.1118/1.598262

[25] M. Fippel, "Fast Monte Carlo Dose Calculation for Photon Beams Based on the VMC Electron Algorithm," Medical Physics, Vol. 26, No. 8, 1999, pp. 1466-1475. http://dx.doi.org/10.1118/1.598676

[26] C. M. Ma, E. Mok, A. Kapur, et al., "Clinical Implementation of a Monte Carlo Treatment Planning System," Medical Physics, Vol. 26, No. 10, 1999, pp. 2133-2143.

[27] H. Shimazaki and I. Nakano, "Radiation Myelopathy and Plexopathy,” Brain and Nerve, Vol. 60, No. 2, 2008, pp. 115-121.

[28] P. J. Keall, J. V. Siebers, R. Jeraj and R. Mohan, “The Effect of Dose Calculation Uncertainty on the Evaluation of Radiotherapy Plans,” Medical Physics, Vol. 27, No. 3, 2000, pp. 478-484. http://dx.doi.org/10.1118/1.598916

[29] R. Jeraj and P. Keall, “The Effect of Statistical Uncertainty on Inverse Treatment Planning Based on Monte Carlo Dose Calculation," Physics in Medicine \& Biology, Vol. 45, No. 12, 2000, pp. 3601-3613. http://dx.doi.org/10.1088/0031-9155/45/12/307

[30] C. M. Ma and J. S. Li, "Dose Specification for Radiation Therapy: Dose to Water or Dose to Medium?” Physics in Medicine \& Biology, Vol. 56, No. 10, 2011, pp. 30733090. http://dx.doi.org/10.1088/0031-9155/56/10/012

[31] B. R. Walters, R. Kramer and I. Kawrakow, "Dose to Medium versus Dose to Water as an Estimator of Dose to Sensitive Skeletal Tissue,” Physics in Medicine \& Biology, Vol. 55, No. 16, 2010, pp. 4535-4546. http://dx.doi.org/10.1088/0031-9155/55/16/S08

[32] C. M. Ma, S. B. Jiang, T. Pawlicki, et al., "A Quality Assurance Phantom for IMRT Dose Verification,” Physics in Medicine \& Biology, Vol. 48, No. 5, 2003, pp. 561572. http://dx.doi.org/10.1088/0031-9155/48/5/301

[33] F. Verhaegen and S. Devic, "Sensitivity Study for CT Image Use in Monte Carlo Treatment Planning," Physics in Medicine \& Biology, Vol. 50, No. 5, 2005, pp. 937-946. http://dx.doi.org/10.1088/0031-9155/50/5/016

[34] M. Bazalova, L. Beaulieu, S. Palefsky and F. Verhaegena, "Correction of CT Artifacts and Its Influence on Monte Carlo Dose Calculations,” Medical Physics, Vol. 34, No. 6, 2007, pp. 2119-2132. http://dx.doi.org/10.1118/1.2736777 